\title{
Photoionization model analysis of the planetary nebula Hu1-2^
}

\author{
S. Hyung ${ }^{1}$, S. R. Pottasch ${ }^{2}$, and W. A. Feibelman ${ }^{3}$ \\ ${ }^{1}$ School of Science Education (Astronomy), Chungbuk National University, 48 Gaeshin-dong Heungduk-gu, CheongJu, \\ ChungBuk 361-763, South Korea \\ e-mail: hyung@chungbuk.ac.kr \\ 2 Kapteyn Astronomical Institute, PO Box 800, 9700 AV Groningen, The Netherlands \\ 3 Laboratory for Astronomy and Solar Physics, Code 681, Goddard Space Flight Center, Greenbelt, MD 20771, USA
}

Received 1 April 2004 / Accepted 9 June 2004

\begin{abstract}
We have obtained high resolution optical spectra of the planetary nebula $\mathrm{Hu}$ 1-2 in the wavelength region of $3700 \AA-10050 \AA$, with the Hamilton Echelle Spectrograph (HES) at Lick Observatory. Diagnostic analysis indicates that the nebular gas can be represented by inhomogeneous shells of electron density $N_{\epsilon} \simeq 4000-10000 \mathrm{~cm}^{-3}$, and a gas temperature of $12000-18000 \mathrm{~K}$. Using the spherically symmetric photoionization model with appropriate abundances, we tried to accommodate the observed physical conditions and high electron temperatures. The chemical composition of the nebula was derived from calculations using a photoionization model which predicts the observed IUE, HES and ISO line intensities; and the composition was then compared to previous determinations. Model analysis confirms the semi-empirically determined abundance derivations carried out in earlier studies. He and $\mathrm{N}$ abundances are high, but those of $\mathrm{C}, \mathrm{O}, \mathrm{Ne}$ and $\mathrm{S}$ are very low.
\end{abstract}

Key words. ISM: abundances - ISM: planetary nebulae: individual: Hu 1-2

\section{Introduction}

The planetary nebula $(\mathrm{PN}) \mathrm{Hu} 1-2$ (PNG 086.5-08.8) is a butterfly-like object of about $6^{\prime \prime} \times 2^{\prime \prime}$ in radio maps. It is classified as a Peimbert's type I, i.e. objects with highly enhanced helium and nitrogen abundances, probably evolved from massive progenitors (see Peimbert \& Torres-Peimbert 1983).

We secured a high dispersion optical spectrum, from 3700 to $10050 \AA$, with the Hamilton Echelle Spectrograph (HES) at the Lick Observatory. Pottasch et al. (2003, hereafter, Paper I) analyzed the ISO far infrared spectrum, combined with the IUE ultraviolet observations to find the nebular abundances. They also used some HES data to derive the abundances. In our high dispersion spectrum, the spectral profiles are well separated into double peaks, so the spectrum of each blue and red component must be investigated separately during the extraction of the diagnostic information, if possible. The photoionization (P-I) model construction would allow us to find even abundances for rare elements. In this study, we will investigate the PN physical conditions and the diagnostics, from the HES high dispersion spectra, with an appropriate P-I model. We will also derive the abundances of $\mathrm{Hu} 1-2$, based on our modelling efforts, and compare them with the previously determined empirical abundances, and with the average PN or solar abundances. With a proper P-I model which best fits the observed line intensities, one can also get an effective

* Table 3 is only available in electronic form at http://www . edpsciences.org
Table 1. Some basic data for Hu 1-2 (PNG 086.5-08.8).

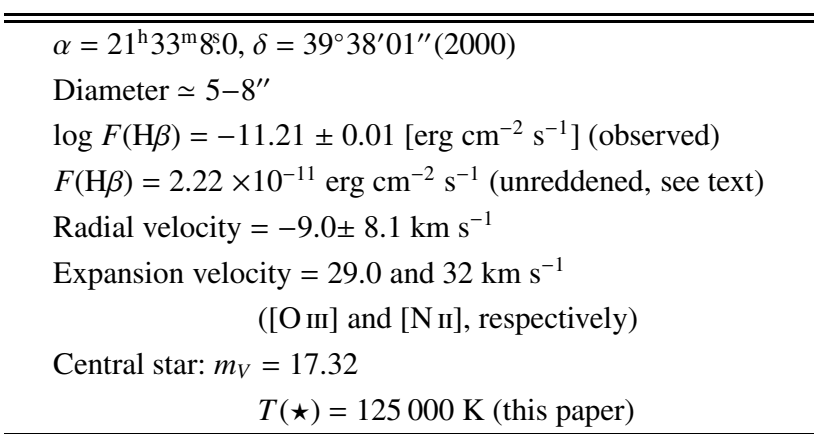

These data are from Acker et al. (1992), unless otherwise indicated.

temperature for the central star of the PN (CSPN), and thereby infer its evolutionary status. We will check whether the PI model can represent the observed electron temperatures and densities. Table 1 gives some basic data for Hu 1-2.

\section{Observations}

The spectrum in the visual wavelength region has been taken with the Hamilton Echelle Spectrograph (HES) at the Coudé focus of the $3 \mathrm{~m}$ Shane telescope of Lick Observatory. The spectral data were taken at 3 positions, centered at positions $\sim 3^{\prime \prime}$ north and $\sim 3^{\prime \prime}$ south of the CSPN and at the center 
Table 2. Observing $\log$ of $\mathrm{Hu} 1-2$.

\begin{tabular}{lcccc}
\hline \hline Date (UT) & Setup & Exp. (m) & Setup & Exp. (m) \\
\hline & & North & & \\
1991 Aug. 31 & S127 & 120 & 123 & 120 \\
& S127 & 15 & & \\
1991 Sep. 1 & S125 & 120 & 121 & 120 \\
& S125 & 25 & & \\
1991 Sep. 2 & S124 & 100 & 126 & 100 \\
2001 Aug. 28, 11 & $2 \mathrm{~K}$ & 60 & & \\
\hline & & Center & & \\
1992 Sep. 16 & S121 & 120 & 127 & 30 \\
1992 Sep. 17 & S125 & 120 & 125 & 5 \\
1992 Sep. 18 & S123 & 120 & & \\
\hline & & South & & \\
1991 Aug. 30 & S121 & 120 & 121 & 10 \\
1991 Aug. 31 & S127 & 15 & 125 & 6 \\
\hline
\end{tabular}

Although the large $2048 \times 2048$ pixel CCD observation in 2001 covered the whole HES echelle pattern, the earlier smaller CCD chip required several settings (see text).

of the image, with a small $800 \times 800$ pixel CCD (on 1991 Aug. and Sep., 1992 Sep.); and these were supplemented by a large $2048 \times 2048$ pixel CCD observation (on 2001 Aug.). The sky was clear during our observations, and seeing was typically $\sim 1.5^{\prime \prime}$ in 1991 and 1992 , and $\sim 0.8^{\prime \prime}$ in 2001. The 2001 observation was carried out only at the north position of the PN image. The slit entrance employed was $\sim 1.2^{\prime \prime} \times 4^{\prime \prime}$ in image size at the Coudé focus, and it was placed at the center of the nebula. The spectral resolution limited by this slit size was about 2 pixels on the CCD chip, which amounted to $0.05 \AA$ wavelength dispersion at $3600 \AA$, and increased to $0.15 \AA$ at $8850 \AA$. The observations with the $800 \times 800$ pixel CCD chip required 6 setups, i.e. S124, S125 and S126 for the long wavelengths; S121 and S123 for the short wavelengths; and S127 for the middle wavelengths, to obtain $\mathrm{H} \alpha$, [O III] and $\mathrm{H} \beta$ with one chip setting. 100 and $120 \mathrm{~min}$ long exposures are necessary to detect faint lines, while 15 and 25 min short exposures are required to avoid possible saturation effects in strong lines. The 2001 observation was done with a large $2048 \times 2048(2 \mathrm{~K})$ pixel CCD, which covered the whole echellogram in a single exposure. More details of the spectrograph used are described in Hyung (1994). We summarize the exposures in Table 2.

For data reduction, we also took exposures of a Th-Ar arc lamp to set the wavelength dispersion scale; a dome-quartz lamp to fix a flat field, which allowed us to correct for pixel-to-pixel sensitivity fluctuations; and finally exposures on standard stars of known energy distribution, i.e. HR 7596 and HR 9087. The reduction procedures are described in Hyung (1994). About 80 strong lines of the Hamilton Echelle data were already presented in Paper I, and these were studied together with the ISO data to derive abundances using the Ionization Correction Factor (ICF) method. In Table 3, we list a fuller set of the normalized line intensities. About 80 additional weak intensity lines are listed. The line intensities are given on the scale of $I(4861)=100.0$, corrected for interstellar extinction, with an extinction coefficient $C=0.60$. Some lines, e.g. N III 4640.66 and He I, are newly measured, but most of the lines also found in Paper I have the same intensity values. Columns 1 and 2 of Table 3 list the measured wavelength (corrected for radial velocity) and the ion. By comparing the observed wavelength, we can find the radial velocity of the nebula as follows: since most spectral lines are separated into double peaks, we derive the radial velocity by averaging the values found for the blue and red components at each slit position: (1) $-1.54 \mathrm{~km} \mathrm{~s}^{-1}$ for the CSPN position (from $-30.71 \pm$ 0.92 (blue) and $27.64 \pm 0.83 \mathrm{~km} \mathrm{~s}^{-1}$ (red)); (2) $-4.39 \mathrm{~km} \mathrm{~s}^{-1}$ for the north position (from $-32.44 \pm 0.97$ (blue) and $23.67 \pm$ $\left.0.91 \mathrm{~km} \mathrm{~s}^{-1}(\mathrm{red})\right)$. These values were obtained from a comparison of the observed wavelengths of the strong lines (I(lines) > 1.0) with the laboratory wavelengths, using the least squares method. From the above values, we adopt the CSPN slit position value, i.e. $-1.54 \pm 0.88 \mathrm{~km} \mathrm{~s}^{-1}$, as the radial velocity of the PN, whereas Acker et al. (1992) quoted $-9.0 \pm 8.1 \mathrm{~km} \mathrm{~s}^{-1}$. This discrepancy is perhaps caused by the slit positions being different. Column 3 gives the large CCD chip data for 2001, while Cols. 4-9 give the earlier smaller CCD chip data. Since most lines are well separated into the red $(R)$ and blue $(B)$ Doppler-shifted peaks, we list separately the combined, the red, \& the blue components, i.e. $I(R+B), I(R)$ and $I(B)$, respectively, all on the scale of $I(4861)=100.0$. The extinction coefficient $C=0.60$ was found from Balmer line ratios, such as $F(\mathrm{H} \alpha) / F(\mathrm{H} \beta)$, and from a comparison of Balmer and Paschen lines of the same upper quantum number. Fuller discussions on the earlier derivations of $C$ can be found in Paper I. In Table 3 we have given 1 or 2 more significant figures than the data justify, to avoid round-off errors.

\section{Nebular diagnostics}

In Paper I, the electron density and temperature were found from the combined data set of IUE, optical and ISO data. Most of the optical line profiles in Table 3 show a double peak feature, which will allow us to find the physical condition, electron density and temperature for the approaching and receding shells, from the blue and red line components. Separate analysis of these components might provide more accurate physical information for the PN.

Figure 1 shows the diagnostics based on the line ratios involving equivalent $\mathrm{p}^{2}$ and $\mathrm{p}^{4}$ electrons. Electronic collision strengths involving the plasma and nebular diagnostics are constantly updated from the most recently available data, as in our previous investigations, e.g. Hyung et al. (2001). The first two figures are for the north slit position: the electron densities are in the range $10^{3.6}-10^{4.0} \mathrm{~cm}^{-3}$ or 4000 $10000 \mathrm{~cm}^{-3}$, but the electron temperatures are strongly scattered. It seems that the lines of high ionization potential are formed in the extremely high temperature region. The diagnostics obtained from the blue line profiles, Fig. 1b, appear to be more complicated or highly scattered than those from the red line profiles: the physical condition of the approaching region of the shell is perhaps more complicated and inhomogeneous than the receding region for the central slit position. 

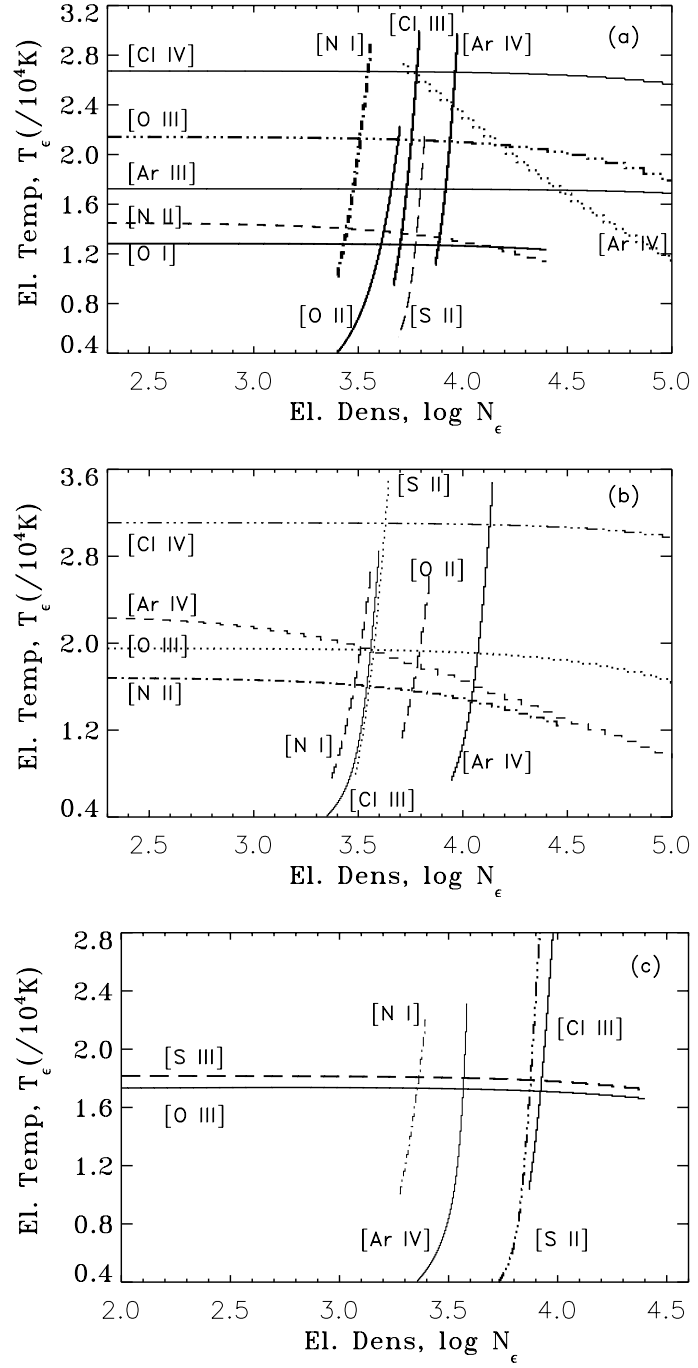

Fig. 1. Diagnostics showing $T_{\epsilon}$ and $N_{\epsilon}$. a) Red profile or receding shell component of North; b) blue profile or approaching shell of North; c) red profile of Center.

There is not much diagnostic information, due to an incomplete observation at this position. Figure 1c shows the diagnostics obtained from the red line profiles, for the center position. Diagnostics of most ions do not converge at any special point. According to Aller \& Liller (1968: see their Fig. 1), the $\mathrm{He}$ II $4686 / \mathrm{H} \beta$ ratio $\sim 0.88$ implies that this $\mathrm{PN}$ has excitation class 4 , and the line ratio $I(\lambda 5007+4959) / \mathrm{I}(\mathrm{H} \beta)$ indicates excitation class 5.5. The electron temperatures of the high excitation lines are extremely high, though. The nebula appears to consist of many inhomogeneous blobs and filaments, and it would be interesting to check whether the P-I model, without shock heating contribution, could produce the precise physical conditions of the shell. Some effects of $T_{\epsilon}$ fluctuation may also exist (see Peimbert et al. 1995).

\subsection{Electron density}

Table 4 lists line ratios suitable for fixing the electron density, $N_{\epsilon}$. The lines are arranged roughly from low to high excitation. The ions and the wavelengths of the lines used to determine
Table 4. Line ratios suitable for fixing $N_{\epsilon}$.

\begin{tabular}{|c|c|c|c|c|}
\hline Ion & $\begin{array}{c}\text { Lines } \\
\text { used }\end{array}$ & $\begin{array}{c}\text { Ratio } \\
(R)\end{array}$ & $\begin{array}{c}\text { Ratio } \\
(B)\end{array}$ & $\begin{array}{l}\log N_{\epsilon} \\
(R, B)^{\dagger}\end{array}$ \\
\hline \multicolumn{5}{|c|}{ North } \\
\hline$\left[\mathrm{N}_{\mathrm{I}}\right]$ & $5198 / 5200$ & 1.53 & 1.54 & $(3.5,3.5)$ \\
\hline$\left[\mathrm{S}_{\text {II }}\right]$ & $6731 / 6716$ & 1.77 & 1.57 & $(3.8,3.6)$ \\
\hline [O II $]$ & $3726 / 3729$ & 2.05 & 2.19 & $(3.7,3.8)$ \\
\hline [Cl III] & $5538 / 5518$ & 1.38 & 1.19 & $(3.7,3.6)$ \\
\hline [Ar IV] & $4740 / 4711$ & 1.38 & 1.60 & $(3.9,4.1)$ \\
\hline \multicolumn{5}{|c|}{ Center } \\
\hline [S II] & $6731 / 6716$ & 1.83 & 2.13 & $(3.9, ?)$ \\
\hline$\left[\mathrm{Cl}_{\mathrm{III}}\right]$ & $5538 / 5518$ & 1.66 & 1.15 & $(3.9,3.5)$ \\
\hline [Ar IV] & $4740 / 4711$ & 1.04 & 0.869 & $(3.5,3.5)$ \\
\hline
\end{tabular}

$(R)$ and $(B)$ : red and blue components, respectively. ${ }^{\dagger} \log N_{\epsilon}\left(\mathrm{cm}^{-3}\right)$ at $T_{\epsilon}=17000 \mathrm{~K}$. "?" means out of range.

$N_{\epsilon}$ are listed in the first two columns, while the observed line intensity ratios are given in Cols. 3 and 4, for the receding and approaching components, respectively; and finally the derived number densities, $\log N_{\epsilon}$, are given in the last column; these values are derived assuming an electron temperature of $T_{\epsilon}=$ $17000 \mathrm{~K}$ (see Table 5). More precise values can, of course, be found from Fig. 1. Although there is no indication of any systematic difference between the rear receding and the front approaching shells, the electron density appears to be in the range between 4000 and $10000 \mathrm{~cm}^{-3}$, with one exception of $N_{\epsilon} \simeq 12500 \mathrm{~cm}^{-3}$ in (Ar IV). If we examine the spectral data of the northern blob only, the density of the high excitation lines appears to be slightly higher than that of the low excitation lines (see Figs. 1a and b). Since the data did not cover the full wavelength range for the central region, there are not very many lines in this region (see Fig. 1c). We will use a density of $5000 \mathrm{~cm}^{-3}$ in deriving the electron temperature in the next section.

\subsection{Electron temperature}

A number of ions sensitive to the electron temperature are listed in Table 5. The successive columns list the ions, the lines used, the corresponding intensity ratios for the red and blue components, and finally the electron temperatures derived. As in Table 4, we list the ions roughly in order of increasing ionization stages, and the electron temperatures are derived with the above-mentioned density, $N_{\epsilon}=5000 \mathrm{~cm}^{-3}$. The electron temperatures appear to increase roughly as a function of ionization potential. This result agrees with the derivation in Paper I. The electron temperatures of most other ions are extremely high, viz. above $17000 \mathrm{~K}$. Similar results are found from the ISO line spectrum (see Paper I). As pointed out in Paper I, the two ions with low ionization potential, $\mathrm{O}_{\text {I }}$ and $\mathrm{N}$ II, which might be formed in the outer regions, show lower electron temperatures, but they are still high compared to those in other PNs, i.e. $13000-15000 \mathrm{~K}$. We examine whether the ordinary photoionization model can predict these much higher electron temperatures. 
Table 5. Line ratios suitable for fixing $T_{\epsilon}$.

\begin{tabular}{lcccc}
\hline \hline Ion & $\begin{array}{c}\text { Lines } \\
\text { used }\end{array}$ & $\begin{array}{c}\text { Ratio } \\
(R)\end{array}$ & $\begin{array}{c}\text { Ratio } \\
(B)\end{array}$ & $\begin{array}{c}T_{\epsilon} / 10^{3} \mathrm{~K} \\
(R, B)^{\dagger}\end{array}$ \\
\hline & North & & & \\
{$\left[\mathrm{O}_{\mathrm{I}}\right]$} & $6300 / 5577$ & 52.5 & - & $(12.7,-)$ \\
{$\left[\mathrm{N}_{\text {III }}\right]$} & $(6548+6584) / 5755$ & 39.3 & 31.2 & $(13.7,15.8)$ \\
{$[\mathrm{Ar}$ III $]$} & $(7751+7136) / 5192:$ & 50.9 & 23.6 & $(17.2,-?-)$ \\
{$\left[\mathrm{O}_{\text {III }}\right]$} & $(4959+5007) / 4363$ & 35.4 & 41.2 & $(21.3,19.3)$ \\
{$\left[\mathrm{S}_{\text {III }}\right]$} & $9069 / 6312^{\ddagger}$ & 4.06 & 4.75 & $(-?-, 29.9)$ \\
{$[\mathrm{Ar}$ IV $]$} & $(4740+4711) / 7171:$ & 32.9 & 44.3 & $(26.5,18.7)$ \\
{$[\mathrm{Cl}$ IV $]$} & $8046 / 5324:$ & $5.73:$ & $5.18:$ & $(26.7,31.0)$ \\
& Center & & & \\
{$\left[\mathrm{N}_{\text {II }}\right]$} & $(6548+6584) / 5755$ & 12.9 & 12.2 & $(-?-,-?-)$ \\
{$\left[\mathrm{O}_{\text {III }}\right]$} & $(4959+5007) / 4363$ & 52.7 & 16.5 & $(17.2,-?-)$ \\
{$\left[\mathrm{S}_{\text {III }}\right]$} & $9069 / 6312^{\ddagger}$ & 7.71 & 6.11 & $(18.0,22.2)$ \\
{$[\mathrm{Cl}$ IV $]$} & $8046 / 5324:$ & 4.71 & 6.07 & $(-?-,-?-)$ \\
\hline
\end{tabular}

† Temperature found assuming $N_{\epsilon}=5000 \mathrm{~cm}^{-3}$. The colon (:) means lines with low intensities are involved. "-?-" means out of range, or too high. "Uncertain due to $\mathrm{H} \alpha$ drip correction.

\section{Abundance derivation with theoretical models}

\subsection{Theoretical models}

The distance of Hu 1-2 is not known, although most values given in the literature range between 1 and $2 \mathrm{kpc}$. Recently, Hajian \& Terzian (1996) gave a lower limit of $1.17 \mathrm{kpc}$, based on radio imaging. As in Paper I, we shall adopt a distance of $1500 \mathrm{pc}$.

The P-I code used here is that of Hyung (1994). It was originally made for studying the PN line intensities for bilaterally symmetrical compact objects, see e.g. Hyung \& Aller (1996). Here, we do not employ an axi-symmetric model geometry, since the nebular image is relatively large and the simple spherically symmetric model seems to fit the observed (slit entrance) line intensities fairly well. To construct a theoretical model, one must know the spectral energy distribution (SED), or else certain other properties, of the CSPN. The SED of the CSPN can be calculated employing a model atmosphere. The hydrogen Zanstra temperature $T_{\mathrm{Z}}(\mathrm{H})$ is about $100000, \mathrm{~K}$ and the ionized helium Zanstra temperature $T_{\mathrm{z}}\left(\mathrm{He}_{\mathrm{II}}\right)$ is about $145000 \mathrm{~K}$ (see Paper I). In Paper I, $T_{\text {star }}=140000 \mathrm{~K}$ is the most probable temperature to explain various high excitation lines in the nebula. We directly applied Hubeny's theoretical model atmospheres (Hubeny 1988), based on some of the selected properties of the CSPN, i.e. $T_{\text {eff }}$, stellar radius, and $\log g$, to the photoionization modelling, until it gave a correct level of nebular excitation (using the energy-balance method and the Zanstra method), and the correct electron temperatures. From our trials, we found that model atmospheres with relatively high temperatures are suitable for the CSPN, e.g. $T_{\text {eff }}=125000 \mathrm{~K}$. The P-I model predictions with a lower temperature model atmosphere, e.g. $T_{\text {eff }} \sim 100000 \mathrm{~K}$, or higher temperature, e.g. $T_{\text {eff }} \sim$ $145000 \mathrm{~K}$, were not satisfactory. The CSPN energy distribution used in the model has $T_{\text {eff }}=125000 \mathrm{~K}$, and $\log g=5.3$,
Table 6. Model details for $\mathrm{Hu}$ 1-2.

\begin{tabular}{ll}
\hline \hline Parameter & Model \\
\hline$R_{\text {in }}$ & $0.017 \mathrm{pc}$ \\
$R_{\text {out }}^{a}$ & $0.024 \mathrm{pc}\left(\sim 3.25^{\prime \prime}\right)$ \\
$N_{\mathrm{H}}$ & $6300 \mathrm{~cm}^{-3}$ \\
& \\
DISTANCE & $1500 \mathrm{pc}$ \\
$M_{\text {dust }} / M_{\text {gas }}$ & 0.001 \\
$F(\mathrm{H} \beta)-$ obs ${ }^{b} \simeq$ & $2.45(-11) \mathrm{erg} \mathrm{cm}^{-2} \mathrm{~s}^{-1}$ \\
$F(\mathrm{H} \beta)-$ pred & $2.65(-11) \mathrm{erg} \mathrm{cm}^{-2} \mathrm{~s}^{-1}$ \\
CSPN $T(\star)^{c}$ & $125000 \mathrm{~K}(\log g=5.3)$ \\
$\quad R(\star)$ & $0.056 R_{\odot}\left(L(\star)=700 L_{\odot}\right)$ \\
$T_{\epsilon}([\mathrm{O}$ II, III, Iv $])$ & $14200,15300,16600 \mathrm{~K}$ \\
Magnitude & $V_{\text {obs }}^{d}=16.05-16.50$ and $V_{\text {pred }}=16.03$ \\
\hline
\end{tabular}

${ }^{a}$ Density bounded. ${ }^{b}$ Extinction corrected with $C=0.6 .{ }^{c}$ Hubeny non-LTE model atmosphere. See text. ${ }^{d}$ Corrected with $E(B-V)=$ 0.41 ( or $C=0.60$ ).

with $\mathrm{He} / \mathrm{H}=0.13$, and with a nebular heavy element distribution in the central star. In Fig. 1, the electron number density is $N_{\epsilon} \sim 4000-10000 \mathrm{~cm}^{-3}$. The nebula is assumed to be a homogeneous shell with $N_{\mathrm{H}}=6300$ (or $\left.N_{\epsilon} \sim 7500\right) \mathrm{cm}^{-3}$. No filling factor was introduced in the shell gas. We assume a central star radius of $R_{*}=0.056 R_{\odot}$, and, as a result, $L_{*}=700 L_{\odot}$. We assumed only a small value for the dust-to-gas ratio, $M_{\mathrm{dust}} / M_{\mathrm{gas}}=$ 0.001 .

The details of the parameters adopted in our model are given in Table 6. For an assumed distance of $1.5 \mathrm{kpc}$, we calculate the CSPN properties and the absolute $\mathrm{H} \beta$ flux. Acker et al. (1992) quoted a visual magnitude for the CSPN of $m_{v}=17.32$, while Heap et al. (1990) measured it to be $m_{v}=17.76$; from this, the intrinsic visual magnitudes are $V_{\mathrm{obs}}=16.05-16.50$, with $C=0.60$ (or $E_{B-V}=0.41$, and the corresponding total extinction $A_{\mathrm{v}}$ is taken as $3.1 E_{B-V}$ ). The model predictions give values that are about one magnitude lower than the observed ones: $V_{\text {pred }}=16.03$ and $B_{\text {pred }}=15.73$. The outer shell boundary is slightly smaller than the observed nebular optical image radius. To fit the observed outer boundary size, we must introduce a filling factor, which was not done.

Table 7 compares the observed and predicted intensities. Intensities for the CSPN and north blob positions obtained in 1991 and 1992 are given in Cols. 3 and 4, respectively, while the north position $2 \mathrm{~K} \mathrm{CCD}$ data taken in 2001 are given in Col. 5. The IUE Archive and ISO intensities are calculated using the flux data taken from Paper I (see their Tables 1 and 3), which are also listed in square brackets or parentheses in Col. 4. Column 6 lists the predicted intensities, and all of the values are given on a scale of $I(\mathrm{H} \beta)=100$.

For most ions, fairly reasonable agreement between the observed and predicted intensities is achieved; but in some cases, especially the weakly detected IUE lines, e.g. N IV] and $\mathrm{N}$ Iv], we find a large discrepancy. As usual, we properly treated collisionally excited contributions in predicting the He lines. The agreement for He I and He II seems to be fine. 
Table 7. Comparison of observed and predicted intensities.

\begin{tabular}{|c|c|c|c|c|c|}
\hline El-ion & $\lambda$ & $I I(\mathrm{C})$ & $I(\mathrm{~N})$ & $I(\mathrm{~N}-2 \mathrm{~K})$ & $I$ I(Model) \\
\hline \multirow[t]{3}{*}{$\mathrm{He}$ I } & 5876 & 11.51 & 10.06 & 7.35 & 9.33 \\
\hline & 6678 & - & 2.44 & 2.41 & 2.09 \\
\hline & 4471 & 3.85 & 2.68 & 3.23 & 3.35 \\
\hline \multirow[t]{3}{*}{ Не II } & 4686 & 88.76 & 73.84 & 81.34 & 83.71 \\
\hline & 5412 & 5.44 & 5.95 & 7.13 & 7.20 \\
\hline & 1640 & & [604] & & 632 \\
\hline \multirow{2}{*}{$\mathrm{C}_{\text {II }}$} & $2325 / 28$ & & [83.8] & & 54.7 \\
\hline & 4267 & & - & & 0.09 \\
\hline $\mathrm{C}_{\text {III }}$ & 1907/09 & & [441] & & 560 \\
\hline $\mathrm{C}_{\mathrm{IV}}$ & $1548 / 51$ & & [712] & & 505 \\
\hline \multirow[t]{3}{*}{$\mathrm{N}_{\text {II }}$} & 6584 & 241.7 & 171.1 & 136.3 & 158.6 \\
\hline & 6548 & 71.76 & 58.96 & 40.98 & 54.75 \\
\hline & 5755 & 5.67 & 5.67 & 4.42 & 6.02 \\
\hline $\mathrm{N}_{\text {III }}$ & $1747-52$ & & [171] & & 134 \\
\hline $\mathrm{N}_{\text {IV }}$ & $1483 / 86$ & & [256] & & 91.7 \\
\hline $\mathrm{Nv}$ & $1239 / 42$ & & [243] & & 28 \\
\hline \multirow[t]{2}{*}{$\mathrm{O}_{\mathrm{I}}$} & 6300 & - & 6.67 & 5.59 & 0.14 \\
\hline & 6363 & 2.81 & 2.12 & 2.07 & 0.05 \\
\hline \multirow[t]{6}{*}{$\mathrm{O}_{\text {II }}$} & 3726 & - & 31.94 & 30.54 & 29.31 \\
\hline & 3729 & 14.94 & 15.45 & 14.42 & 12.68 \\
\hline & $7321 / 2$ & 4.24 & - & 4.55 & 3.91 \\
\hline & $7332 / 3$ & 3.18 & - & 3.97 & 3.13 \\
\hline & $7321 / 2$ & - & - & 4.55 & 3.91 \\
\hline & $7332 / 3$ & - & - & 3.97 & 3.13 \\
\hline \multirow[t]{4}{*}{$\mathrm{O}_{\text {III }}$} & $1660 / 66$ & & [45] & & 39 \\
\hline & 4363 & 24.57 & 25.65 & 19.19 & 14.86 \\
\hline & 4959 & 279.0 & 221.4 & 224.4 & 254.1 \\
\hline & 5007 & 823.3 & 717.0 & 745.7 & 731.9 \\
\hline OIV & $1397-1407$ & & [57] & & 14 \\
\hline \multirow[t]{2}{*}{$\mathrm{Ne}$ III } & 3868 & 86.90 & 70.27 & 68.67 & 81.62 \\
\hline & 3968 & 27.57 & 26.37 & 25.83 & 24.36 \\
\hline \multirow[t]{2}{*}{ Ne IV } & $2422 / 25$ & & [213] & & 50 \\
\hline & $4725 / 27$ & 2.23 & 2.96 & - & 0.53 \\
\hline $\mathrm{Nev}$ & 3426 & & (181) & & 14.47 \\
\hline \multirow[t]{4}{*}{$\mathrm{S}_{\text {II }}$} & 4068 & 3.41 & 3.30 & 2.99 & 1.48 \\
\hline & 4076 & 0.99 & 1.19 & - & 0.50 \\
\hline & 6717 & 6.17 & 5.42 & 4.78 & 1.30 \\
\hline & 6731 & 11.92 & 11.02 & 7.47 & 2.39 \\
\hline \multirow[t]{3}{*}{$\mathrm{S}_{\text {III }}$} & 6312 & 2.14 & 3.48 & 3.24 & 1.88 \\
\hline & 9069 & 14.86 & 13.38 & 12.26 & 18.96 \\
\hline & 9531 & 36.97 & 21.54 & 25.24 & 46.20 \\
\hline S IV & $10.5 \mu \mathrm{m}$ & & (50.8) & & 51.98 \\
\hline $\mathrm{Cl}_{\text {II }}$ & 8579 & - & 0.16 & - & 0.17 \\
\hline \multirow[t]{2}{*}{$\mathrm{Cl}_{\text {III }}$} & 5518 & 0.50 & 0.48 & - & 0.49 \\
\hline & 5538 & 0.71 & 0.59 & 0.59 & 0.78 \\
\hline \multirow[t]{2}{*}{$\mathrm{Cl}_{\text {IV }}$} & 7530 & 0.48 & 0.46 & - & 0.35 \\
\hline & 8046 & 0.84 & 0.87 & 0.90 & 0.87 \\
\hline \multirow[t]{3}{*}{ Ar III } & 5192 & 0.25 & 0.29 & - & 0.20 \\
\hline & 7136 & 11.81 & 10.96 & 9.63 & 12.86 \\
\hline & 7751 & - & 2.18 & 2.67 & 3.11 \\
\hline \multirow[t]{5}{*}{ Ar IV } & 4711 & 5.65 & 4.25 & 5.76 & 4.40 \\
\hline & 4740 & 5.45 & 6.51 & 6.26 & 5.92 \\
\hline & 7238 & 0.37 & 0.37 & - & 0.16 \\
\hline & 7263 & - & 0.31 & - & 0.18 \\
\hline & 7171 & - & 0.28 & - & 0.22 \\
\hline \multirow[t]{2}{*}{$\operatorname{Arv}$} & 6435 & - & 1.32 & 1.86 & 1.28 \\
\hline & 7005 & - & 2.69 & 4.16 & 2.74 \\
\hline $\mathrm{Cav}$ & 5310 & 0.13 & 0.08 & 0.17 & - \\
\hline K IV & 6102 & 0.30 & 0.30 & 0.35 & - \\
\hline Si III & $1883 / 92$ & & [37] & & 36.5 \\
\hline
\end{tabular}

$I(\mathrm{C})$ : slit position at the CSPN position. $I(\mathrm{~N})$ : slit at the north of the CSPN. $I(\mathrm{~N}-2 \mathrm{~K}): 20012 \mathrm{~K}$-data. Intensities in square brackets and parentheses are from Pottasch et al. (2003).
The predictions for $\mathrm{C}$ also seem to be fine, except for the recombination C II $\lambda 4267$ line, and C IV. Simultaneously fitting the optical and $I U E$ nitrogen line intensities with the P-I model seems to be impossible. There may be an unavoidably large error involved in the IUE line intensities: it could be due to measurement uncertainty, or to an overestimated extinction correction. Here, our model predictions are consistent with the optical lines.

Predictions for the ions of $\mathrm{O}, \mathrm{Ne}, \mathrm{S}, \mathrm{Cl}$ and $\mathrm{Ar}$ seem to be generally successful. The prediction for the [O $\mathrm{I}]$ and $\left[\mathrm{S}_{\mathrm{II}}\right]$ lines, which might have formed in or near the neutral cloud region, indicate some problem, though. However, the agreement for oxygen and sulfur lines, including the [S IV] ISO IR line, appears to be fine. Rare elements like $\mathrm{Ca}, \mathrm{K}$, and $\mathrm{Si}$ are all represented by single ionization stages: calcium by [Cav], potassium by [KIV], and silicon by Si III]. Hence, agreement for these ions can be assured, and the abundances of these elements can be found from the model.

The diagnostic electron temperatures are $T_{\epsilon} \sim 15000$, $17000 \mathrm{~K}$ and $20800 \mathrm{~K}$, for [N II], [Ar III] and [O III], respectively (see Fig. 1a and Table 5), while the model predicts $T_{\epsilon} \simeq$ $14200 \mathrm{~K}, 15900 \mathrm{~K}$ and $16600 \mathrm{~K}$, respectively; and it gives $T_{\epsilon} \simeq 14200 \mathrm{~K}$ and $15300 \mathrm{~K}$, for [O II] and [O IV], respectively. The photoionization model cannot predict the large scatter indicated by the diagnostics. The relatively higher temperatures implied by the diagnostics in [O III], [Ar IV] and [Cl IV] are obviously not confirmed by the model. We do not have a reliable way of estimating the error, but there could be other factors or physical mechanisms involved, such as shock heating which may have contributed to the excitation of lines. Since our model structure is an idealized, simple, homogeneous shell, it does not admit a point-to-point fluctuation of $N_{\epsilon}$ and $T_{\epsilon}$. Nonetheless, our photoionization model broadly reproduces the observables. It gives a reasonable effective temperature for the CSPN, similar to derivations by other methods. The CSPN temperature of Hu 1-2 is likely to be around $T_{\text {eff }}=125000 \mathrm{~K}$, similar to that of IC 5117 (see Hyung et al. 2001).

\subsection{Elemental abundances}

Table 8 compares the abundances derived in this paper with earlier values found in Paper I and in the literature. The earlier abundance determinations are mostly obtained from an Ionization Correction Factor (ICF) method. See Paper I for detailed information on these earlier derivations. If we compare our model abundances with the semi-empirical derivation of Paper I, there is a fairly good agreement for $\mathrm{He}, \mathrm{C}, \mathrm{N}, \mathrm{Ar}$ and $\mathrm{Cl}$, i.e. to within $10 \%$, while the currently derived $\mathrm{O}, \mathrm{S}$ and $\mathrm{Ne}$ abundances are $17-40 \%$ lower than those of Paper I. The helium abundances in both this paper and Paper I, are lower than those in Aller \& Czyzak (1983) or Malkov (1998). We adopt 0.13 as the relative helium abundance. Compared with the earlier studies, the uncertain ICF was very small in Paper I, usually less than a factor 1.5 , so the elemental abundances of Paper I are probably the best-determined. Thus, if the present value is close to that in Paper I, we adopt the Paper I value as a recommended elemental abundance relative to $\mathrm{H}^{+}$, 
Table 8. Comparison of abundances in Hu 1-2.

\begin{tabular}{lccccc}
\hline \hline El. & Present & Paper I & AC(1) & Malkov(2) & PT(3) \\
\hline $\mathrm{He}$ & 0.130 & 0.127 & 0.158 & 0.154 & 0.147 \\
$\mathrm{C}(-4)$ & 1.6 & 1.62 & 1.2 & 1.2 & \\
$\mathrm{~N}(-4)$ & 1.7 & 1.9 & 3.2 & 1.5 & 2.2 \\
$\mathrm{O}(-4)$ & 1.3 & 1.57 & 1.1 & 1.1 & 1.6 \\
$\mathrm{~S}(-6)$ & 3.0 & 4.2 & 3.0 & 7.8 & $3.1 *$ \\
$\mathrm{Ar}(-6)$ & 1.2 & 1.1 & 1.6 & 0.87 & 0.79 \\
$\mathrm{Ne}(-5)$ & 3.0 & 4.9 & 6.7 & 5.9 & 3.6 \\
$\mathrm{Cl}(-7)$ & 1.2 & 1.1 & 1.5 & & \\
$\mathrm{Ca}(-8)$ & 9.0 & & & & \\
$\mathrm{~K}(-8)$ & 9.0 & & & & \\
$\mathrm{Si}(-6)$ & 5.0 & & & & \\
\hline
\end{tabular}

(1) Aller \& Czyzak (1983); (2) Malkov (1998); (3) Peimbert \& Torres-Peimbert (1995); * Sabbadin et al. (1987).

$N(\mathrm{el}) / N\left(\mathrm{H}^{+}\right)$. The values are $1.6(-4)$ for $\mathrm{C}$; $1.8(-4)$ for $\mathrm{N}$; 1.1(-6) for Ar; and 1.1(-7) for $\mathrm{Cl}$, respectively. For other uncertain cases, we adopt the mean value from the two papers, but sometimes we chose the value close to either the present model, or to the Paper I value, in the case where derivations from other sources are much closer to one of them, i.e. 1.3(-4) for $\mathrm{O} ; 4.9(-5)$ for $\mathrm{Ne}$; and 3.5(-6) for $\mathrm{S}$.

\section{Conclusions}

Table 9 compares the $\mathrm{Hu}$ 1-2 abundances with the average PN abundances by Aller \& Czyzak (1983) and by Kingburgh \& Barlow (1994), and with the solar abundances given in the literatures. Of a number of nebulae whose ICF abundances had been determined with the high dispersion spectroscopic or ISO measurements, $\mathrm{Hu}$ 1-2 has the lowest abundance yet found (see Paper I). He and $\mathrm{N}$ are exceptions. Except for these two elements, the abundances are even lower than the solar values. The presently derived values for the model are even slightly lower than those in Paper I.

The lower abundances of carbon, oxygen, neon, sulfur and argon, which are not produced in the CSPN in the course of its evolution, and instead reflect the abundances in the star at the time of its formation, suggest that $\mathrm{Hu} \mathrm{1-2} \mathrm{is} \mathrm{much} \mathrm{older}$ than the other PNs observed. As mentioned in Paper I, it also indicates that the CSPN progenitor must be a low mass star, and, as a result, its evolution should have proceeded much more slowly. Meanwhile, a higher abundance of nitrogen and an enhanced abundance of helium, which must have been formed in the course of the evolution of the central star, suggest that it may have been of high mass $\left(\geq 2.4 M_{\odot}\right.$ according to Peimbert $\&$ Torres-Peimbert 1983). Since all the other elemental abundances are very low, it has perhaps not evolved from such a high mass star. Hu 1-2 may still be classified as a Peimberts' type I, though, in view of the enhancement of the He and $\mathrm{N}$ abundances.
Table 9. Comparison of abundances in Hu 1-2.

\begin{tabular}{lrrr}
\hline \hline Element & Hu 1-2 & PN Ave. $^{a}$ & Sun $^{b}$ \\
\hline $\mathrm{He}$ & 0.13 & 0.11 & 0.086 \\
$\mathrm{C}(-4)$ & 1.6 & 6.48 & 2.57 \\
$\mathrm{~N}(-4)$ & 1.8 & 1.4 & 0.63 \\
$\mathrm{O}(-4)$ & 1.3 & 4.93 & 4.57 \\
$\mathrm{Ne}(-5)$ & 4.9 & 12.5 & 6.92 \\
$\mathrm{~S}(-6)$ & 3.5 & 8.08 & 21.4 \\
$\mathrm{Ar}(-6)$ & 1.1 & 2.42 & 1.51 \\
$\mathrm{Cl}(-7)$ & 1.1 & 1.66 & 3.16 \\
$\mathrm{~K}(-8)$ & 9.0 & & 13.2 \\
$\mathrm{Si}(-6)$ & 5.0 & & 35.5 \\
$\mathrm{Ca}(-8)$ & 9.0 & & 229 \\
\hline
\end{tabular}

${ }^{a}$ Average (or normal) abundances from Kingsburgh \& Barlow (1994) and Aller \& Czyzak (1983). ${ }^{b}$ Solar abundances are from ChristensenDalsgaard (1998) for He; Asplund (2003) for C \& N; Asplund et al. (2004) for O, Ne \& Ar; and Grevesse \& Sauval (1998) for others, respectively.

It would be interesting to see what would be the CSPN mass, based on the current model: taking $L(\star)$ and $T(\star)$ at their face values (see Table 6), and utilizing Schönberner's (1983) and Vassiliadis \& Woods (1994) evolutionary tracks, we find a CSPN mass of about $0.56 M_{\odot}$. In addition, these evolutionary tracks suggest a corresponding age of about 22000 years, for the evolution from the AGB progenitor phase. We employed the spherically symmetric P-I model to study the spectra secured from the UV-optical-IR wavelength region. The P-I model constructed in this paper, gives a fairly good representation of the observed physical conditions of $\mathrm{Hu} 1-2$. It confirms that the lower abundances, except for $\mathrm{He}$ and $\mathrm{N}$, are the main cause of the extremely high electron temperatures found in this object. In its main sequence phase, the progenitor star must have been slightly less massive than our Sun, which confirms the Paper I result. As indicated in Paper I, Hu 1-2 could have evolved from a star which had been formed earlier in the history of the evolution of our Galaxy. The Hu 1-2 YSO progenitor might have been formed in a chemically uncontaminated region of the Galaxy, e.g. near the Galactic halo.

Acknowledgements. We express our gratitude to the late Prof. Lawrence H. Aller (UCLA) for his support of this work. He worked with one of the authors, S.H., but unfortunately passed away before he could review a draft of this paper. We thank Dr. André B. Fletcher (Korea Astronomy Observatory) for his proof-reading and Dr. Silvia Torres-Peimbert (UNAM) for her careful review and valuable comments. We are also thankful to the technical staff of Lick Observatory, who helped us secure the Hamilton Echelle data. This work was the result of research activities of the Astrophysical Research Center for the Structure and Evolution of the Cosmos (ARCSEC), supported by the Korea Science \& Engineering Foundation. 


\section{References}

Acker, A., Ochsenbein, F., Stenholm, B., et al. 1992, Strasbourg-ESO Catalogue of Galactic Planetary Nebulae, Garchung bei Munchen, European Southern Observatory

Aller, L. H., \& Czyzak, S. J. 1983, ApJS, 51, 211 (AC83)

Aller, L. H., \& Liller, W. 1968, in Nebulae and Interstellar Matter, ed. B. M. Middlehurst, \& L. H. Aller (Chicago: University of Chicago Press), Chap. 9, 498

Asplund, M. 2003, in CNO in the Universe, ed. C. Charbonnel, D. Schaerer, \& G. Meynet, ASP Conf. Ser., 304, 275

Asplund, M., Grevesse, N., Sauval, A. J., et al. 2004, A\&A, 417, 751

Christensen-Dalsgaard, J. 1998, Space Sci., 85, 19

Grevesse, N., \& Sauval, A. J. 1998, Space Sci. Rev., 85, 161

Hajian, A. R., \& Terzian, Y. 1996, PASP, 108, 258

Heap, S. R., Corcoran, M., Hintzen, P., \& Smith, E. 1990, From Miras to PN, ed. M. O. Mennessier, \& A. Omont (Gif-sur-Yvette: Éditions Frontières)
Hubeny, I. 1988, Computer Phys. Comm., 52, 103

Hyung, S. 1994, ApJS, 90, 119

Hyung, S., Aller, L. H., Feibelman, W. A., \& Lee, S.-J. 2001, ApJ, 563,889

Kingsburgh, R. L., \& Barlow, M. J. 1994, MNRAS, 271, 257

Malkov, Yu. F. 1998, A. Rep., 42, 293

Peimbert, M., Luridiana, V., \& Torres-Peimbert, S. 1995, RMxA\&A, 31,147

Peimbert, M., \& Torres-Peimbert, S. 1983, IAU Symp., 103, 233

Pottasch, S. R., Hyung, S., Aller, L. H., et al. 2003, A\&A, 401, 205 (Paper I)

Sabbadin, F., Cappellaro, E., \& Turatto, M. 1987, A\&A, 182, 305

Schönberner, B. 1983, ApJ, 272, 708

Vassiliadis, E., \& Wood, P. R. 1994, ApJS, 92, 125 


\section{Online Material}


S. Hyung et al.: P-I Model of PN Hu 1-2, Online Material p 2

Table 3. Optical Spectrum of Hu 1-2 (North \& Center).

\begin{tabular}{|c|c|c|c|c|c|c|c|c|}
\hline \multirow[b]{2}{*}{$\lambda_{\mathrm{obs}}$} & \multirow[b]{2}{*}{ Ion } & \multicolumn{3}{|c|}{ "North } & \multirow[b]{2}{*}{$I(B)$} & \multicolumn{3}{|c|}{ Center } \\
\hline & & $I(2001)$ & $I(R+B)$ & $I(R)$ & & $I(R+B)$ & $I(R)$ & $I(B)$ \\
\hline 3705.05 & $\mathrm{He}_{\mathrm{I}}$ & & & 1.01 & & & & \\
\hline 3711.87 & $\mathrm{H}_{\mathrm{I}}$ & & & 1.01 & & & & \\
\hline 3726.07 & {$[\mathrm{O} \mathrm{II}]$} & 30.54 & 31.94 & 39.12 & 12.38 & & & \\
\hline 3728.83 & [O II] & 14.42 & 15.45 & 19.05 & 5.65 & 14.94 & 12.35 & 19.22 \\
\hline 3734.33 & $\mathrm{H}_{\mathrm{I}}$ & & 1.47 & 1.55 & 1.26 & & & \\
\hline 3750.05 & $\mathrm{H}_{\mathrm{I}}$ & & & 2.20 & & & 2.32 & \\
\hline 3759.79 & $\mathrm{O}_{\text {III }}$ & & 1.93 & 2.21 & 1.14 & 2.14 & 1.75 & 2.80 \\
\hline 3797.83 & $\mathrm{H} \mathrm{I}_{\mathrm{I}}$ & & & 3.46 & & 4.68 & 4.15 & 5.57 \\
\hline 3819.61 & $\mathrm{He}_{\mathrm{I}}$ & & 0.36 & & & & & \\
\hline 3835.23 & $\mathrm{H} \mathrm{I}_{\mathrm{I}}$ & 6.79 & 5.30 & & & 6.66 & 5.82 & 8.04 \\
\hline 3857.84 & He II & & 0.37 & & & & & \\
\hline 3862.32 & Si II & & 0.29 & & & & & \\
\hline 3868.74 & {$[\mathrm{Ne}$ III] } & 68.67 & 70.27 & 84.67 & 30.77 & 86.90 & 73.02 & 109.8 \\
\hline 3888.68 & $\mathrm{He}_{\mathrm{I}}$ & 21.86 & & & & 18.89 & & \\
\hline 3889.21 & $\mathrm{H}_{\mathrm{I}}$ & & & 3.42 & & & & \\
\hline 3964.75 & $\mathrm{He}_{\mathrm{I}}$ & & & 0.50 & & 0.49 & 0.33 & 0.75 \\
\hline 3967.45 & {$[\mathrm{Ne}$ III] } & 25.83 & 21.68 & 26.37 & 9.08 & 27.57 & 22.74 & 35.55 \\
\hline 3970.01 & $\mathrm{H} \epsilon$ & 16.97 & 11.55 & 12.29 & 9.48 & 16.24 & 14.91 & 18.45 \\
\hline 4024.78 & He II & & 0.34 & 0.31 & 0.40 & & & \\
\hline 4026.22 & He I (18) & 2.33 & 1.79 & 1.57 & 2.36 & 1.06 & 0.94 & 1.25 \\
\hline 4068.63 & {$\left[\mathrm{~S}_{\mathrm{II}}\right]$} & 2.99 & 3.30 & 4.00 & 1.41 & 3.41 & 2.74 & 4.52 \\
\hline 4076.36 & {$\left[\mathrm{~S}_{\mathrm{II}}\right]$} & & 1.19 & 1.41 & 0.59 & 0.99 & 0.70 & 1.46 \\
\hline 4097.17 & $\mathrm{~N}_{\text {III }}, \mathrm{O}_{\text {II }}$ & & & 0.77 & & 0.75 & 0.64 & 0.95 \\
\hline 4099.87 & $\mathrm{He}_{\text {II }}$ & & 0.79 & 0.74 & 0.95 & 0.96 & & \\
\hline 4101.68 & $\mathrm{H} \delta$ & 23.51 & 20.01 & 21.15 & 16.00 & 25.49 & 23.47 & 28.82 \\
\hline 4103.30 & $\mathrm{~N}_{\text {III }}$ & & 0.39 & 0.43 & 0.30 & & & \\
\hline 4120.66 & $\mathrm{He}_{\mathrm{I}}$ & & 0.28 & & & & & \\
\hline 4143.76 & $\mathrm{He}_{\mathrm{I}}$ & & 0.23 & 0.25 & 0.19 & & & \\
\hline 4163.00 & {$[\mathrm{~K} \mathrm{v}]$} & & & 0.16 & & & & \\
\hline 4199.66 & He II & 1.33 & 1.19 & 1.11 & 1.38 & 1.48 & 1.20 & 1.95 \\
\hline 4227.41 & {$[\mathrm{Fe} \mathrm{v}]$} & & 0.20 & 0.17 & 0.30 & & & \\
\hline 4338.52 & He II & 3.19 & 2.34 & 2.17 & 2.82 & & & \\
\hline 4340.43 & $\mathrm{H} \gamma$ & 43.58 & 49.13 & 48.79 & 49.81 & 48.19 & 47.49 & 49.37 \\
\hline 4357.91 & $\mathrm{O}_{\text {II }}$ & 1.43 & & & & & & \\
\hline 4363.16 & [O III] & 19.19 & 25.65 & 27.61 & 20.28 & 24.57 & 22.34 & 28.25 \\
\hline 4387.93 & $\mathrm{He}_{\mathrm{I}}$ & & 0.35 & 0.39 & 0.26 & & & 1.33 \\
\hline 4471.51 & $\mathrm{He}_{\mathrm{I}}$ & 3.23 & 2.68 & 4.57 & 2.45 & 3.85 & 3.53 & 4.38 \\
\hline 4541.43 & He II & 3.19 & 3.14 & 3.19 & 2.97 & 2.94 & 2.95 & 2.92 \\
\hline 4571.22 & {$[\mathrm{Mg} \mathrm{I}]$} & & 0.34 & 0.46 & 0.29 & 0.34 & 0.28 & 0.44 \\
\hline 4606.12 & $\mathrm{Fe}$ III & & 0.14 & 0.12 & 0.17 & & & \\
\hline 4625.08 & {$[\mathrm{Ar} v]$} & & & & & 0.54 & & \\
\hline 4634.09 & $\mathrm{~N}_{\text {III }}$ & & 0.39 & 0.46 & 0.21 & 0.35 & 0.27 & 0.49 \\
\hline 4640.66 & $\mathrm{~N}_{\text {III }}$ & 0.87 & 0.84 & 0.91 & 0.40 & 1.04 & 0.97 & 1.16 \\
\hline 4641.79 & O III, N III & & & 0.02 & & & & \\
\hline 4647.35 & $\mathrm{C}_{\text {III }}$ & & 0.11 & & & 0.34 & & \\
\hline 4649.14 & $\mathrm{O}_{\text {II }}$ & & 0.04 & & & & & \\
\hline 4651.05 & $\mathrm{O}_{\mathrm{II}}$ & & 0.10 & & & & & \\
\hline 4658.21 & $\mathrm{C}_{\text {IV }},[\mathrm{Fe}$ III] & & 0.22 & 0.21 & 0.24 & 0.28 & 0.28 & 0.29 \\
\hline 4685.58 & $\mathrm{He}_{\text {II }}$ & 81.34 & 73.84 & 74.52 & 71.66 & 88.76 & 87.65 & 90.60 \\
\hline 4711.28 & [Ar IV] & 5.76 & 4.53 & 4.43 & 4.77 & 5.65 & 4.98 & 6.77 \\
\hline 4714.15 & {$[\mathrm{Ne} \mathrm{IV}]$} & 1.02 & 1.40 & 0.84 & 3.02 & 0.80 & 0.81 & 0.79 \\
\hline 4724.06 & {$[\mathrm{Ne} \mathrm{IV}]$} & & 1.36 & 1.32 & 1.45 & 1.21 & 1.08 & 1.42 \\
\hline 4725.47 & {$[\mathrm{Ne}$ iv] } & & 1.61 & 1.52 & 1.86 & 1.02 & 0.83 & 1.33 \\
\hline 4740.09 & [Ar IV] & 6.26 & 6.51 & 6.11 & 7.62 & 5.45 & 5.19 & 5.88 \\
\hline 4859.08 & He II & & 4.59 & 4.49 & 5.52 & 3.02 & & \\
\hline 4861.29 & $\mathrm{H} \beta$ & 100.0 & 100.0 & 100.0 & 100.0 & 100.0 & 100.0 & 100.0 \\
\hline 4922.00 & $\mathrm{He}_{\mathrm{I}}$ & & 0.90 & 1.38 & 0.75 & & 0.99 & \\
\hline 4931.28 & [O III] & & 0.39 & 0.20 & 0.90 & & & \\
\hline
\end{tabular}


S. Hyung et al.: P-I Model of PN Hu 1-2, Online Material p 3

Table 3. continued.

\begin{tabular}{|c|c|c|c|c|c|c|c|c|}
\hline \multirow[b]{2}{*}{$\lambda_{\mathrm{obs}}$} & \multirow[b]{2}{*}{ Ion } & \multicolumn{3}{|c|}{ North } & \multirow[b]{2}{*}{$I(B)$} & \multicolumn{3}{|c|}{$\overline{\text { Center }}$} \\
\hline & & $I(2001)$ & $I(R+B)$ & $I(R)$ & & $I(R+B)$ & $I(R)$ & $I(B)$ \\
\hline 4958.99 & {$[\mathrm{O}$ III] } & 244.4 & 221.4 & 241.6 & 166.4 & 279.0 & 303.5 & 238.5 \\
\hline 4972.14 & {$[\mathrm{Fe} \mathrm{vI}]$} & & 0.20 & & & & & \\
\hline 4987.98 & [Fe VII] & & 0.14 & & & & & \\
\hline 5006.88 & {$[\mathrm{O}$ III] } & 745.7 & 717.0 & 735.4 & 669.2 & 823.3 & 874.6 & 738.6 \\
\hline 5015.81 & $\mathrm{He}_{\mathrm{I}}$ & 1.21 & 2.24 & 2.42 & 1.71 & 1.63 & 1.62 & 1.65 \\
\hline 5017.56 & $\dagger$ & 0.57 & 0.71 & 0.71 & 0.74 & 0.40 & 0.28 & 0.60 \\
\hline 5047.92 & $\mathrm{He}_{\mathrm{I}}$ & & 0.27 & 0.27 & 0.26 & & & \\
\hline 5131.32 & $\mathrm{C}_{\text {III }}$ & & 0.23 & & & & & \\
\hline 5145.51 & {$[\mathrm{Fe} \mathrm{vI}]$} & & 0.20 & 0.09 & 0.22 & 0.19 & 0.13 & 0.29 \\
\hline 5158.47 & [Fe II,VII?] & & 0.25 & & & & 0.25 & \\
\hline 5191.78 & [Ar III] & & 0.29 & 0.29 & 0.37 & 0.25 & 0.27 & 0.23 \\
\hline 5198.10 & {$\left[\mathrm{~N}_{\mathrm{I}}\right]$} & 1.03 & 1.70 & 1.90 & 1.14 & 1.45 & 1.38 & 1.57 \\
\hline 5200.47 & {$\left[\mathrm{~N}_{\mathrm{I}}\right]$} & & 1.11 & 1.24 & 0.74 & 0.96 & 0.96 & 0.96 \\
\hline 5270.58 & {$[\mathrm{Fe}$ III] } & & 0.08 & & & & & \\
\hline 5308.79 & {$[\mathrm{Ca} \mathrm{v}]$} & & 0.17 & 0.20 & 0.16 & 0.08 & & \\
\hline 5323.05 & {$[\mathrm{Cl} \mathrm{IV}]$} & & 0.11 & 0.15 & 0.17 & 0.16 & 0.17 & 0.15 \\
\hline 5334.67 & {$[\mathrm{Fe} \mathrm{II}]$} & & 0.14 & & & 0.19 & 0.14 & 0.29 \\
\hline 5411.30 & He II & 7.13 & 5.95 & 6.44 & 5.34 & 5.44 & 5.98 & 4.54 \\
\hline 5460.39 & line? & 0.69 & & & & & & \\
\hline 5517.73 & {$[\mathrm{Cl}$ III] } & & 0.48 & 0.48 & 0.43 & 0.50 & 0.44 & 0.60 \\
\hline 5537.89 & [Cl III] & 0.59 & 0.59 & 0.66 & 0.51 & 0.71 & 0.73 & 0.69 \\
\hline 5577.18 & {$\left[\mathrm{O}_{\mathrm{I}}\right]$} & & & 0.16 & & & & \\
\hline 5591.79 & $\mathrm{O}_{\text {III }}$ & & & 0.17 & & & 0.19 & \\
\hline 5592.06 & $\mathrm{O}_{\text {III }}$ & & 0.13 & 0.12 & & & & \\
\hline 5630.40 & {$[\mathrm{Fe}$ vI $]$} & & 0.08 & & & & & \\
\hline 5676.78 & {$[\mathrm{Fe}$ vI $]$} & & 0.18 & & & & & \\
\hline 5697.09 & line? & & & & & 0.21 & & \\
\hline 5720.52 & {$[\mathrm{Fe}$ VII $]$} & & 0.35 & & & 0.31 & 0.31 & 0.31 \\
\hline 5754.71 & {$[\mathrm{~N}$ II $]$} & 4.42 & 5.67 & 7.22 & 2.82 & 5.67 & 6.12 & 4.93 \\
\hline 5768.52 & $\dagger$ & & & & & 0.17 & & \\
\hline 5791.98 & He II? & & & & & 0.22 & & \\
\hline 5800.28 & $\mathrm{C}_{\text {IV }}$ & & 0.14 & & & & & \\
\hline 5812.52 & $\mathrm{C}_{\text {IV }}$ & & 0.11 & & & & & \\
\hline 5843.96 & line? & & & & & 0.06 & & \\
\hline 5846.26 & He II & & & & & 0.08 & & \\
\hline 5856.76 & He II & & 0.10 & & & & & \\
\hline 5875.75 & $\mathrm{He}_{\mathrm{I}}$ & 7.35 & 10.06 & 11.73 & 4.85 & 11.51 & 12.67 & 9.58 \\
\hline 5881.95 & $\mathrm{He}$ II & & 0.10 & & & & & \\
\hline 5896.41 & He II & & & & & 0.28 & & \\
\hline 5913.13 & He II & & 0.07 & & & & & 0.41 \\
\hline 5931.27 & $\mathrm{~N}_{\text {II }}$ & & & & & 0.15 & & \\
\hline 5952.77 & He II & & 0.21 & 0.15 & 0.30 & 0.13 & & \\
\hline 5976.63 & He II & & 0.13 & 0.14 & & 0.13 & & \\
\hline 6004.63 & He II & & 0.12 & & & & 0.21 & \\
\hline 6036.36 & He II & & 0.17 & 0.08 & & 0.19 & & \\
\hline 6074.04 & He II & & 0.18 & 0.20 & 0.24 & 0.23 & & \\
\hline 6086.16 & {$[\mathrm{Cav}]+[\mathrm{FeviI}]$} & & 0.52 & & & & 0.67 & \\
\hline 6101.59 & {$[\mathrm{~K}$ IV $]$} & & 0.35 & 0.26 & 0.27 & 0.30 & 0.28 & 0.35 \\
\hline 6117.88 & He II & & 0.17 & 0.23 & 0.29 & & & \\
\hline 6170.49 & He II & & 0.23 & 0.17 & 0.16 & 0.22 & & \\
\hline 6227.68 & {$[\mathrm{~K}$ vI $]$} & & 0.23 & & & 0.10 & & \\
\hline 6233.49 & $\mathrm{He}$ II & & 0.29 & 0.22 & 0.30 & & 0.45 & \\
\hline 6300.38 & {$\left[\mathrm{O}_{\mathrm{I}}\right]$} & 5.59 & 6.67 & 8.49 & 1.69 & & & \\
\hline 6310.99 & He II & & & & 0.22 & & & \\
\hline 6312.00 & [S III] & 3.24 & 2.76 & 3.48 & 2.37 & 2.14 & 1.80 & 2.70 \\
\hline 6363.64 & {$\left[\mathrm{O}_{\mathrm{I}}\right]$} & 2.07 & 2.12 & 2.72 & 0.84 & 2.81 & 2.80 & 2.82 \\
\hline 6406.18 & He II & & 0.34 & 0.40 & 0.41 & & 0.61 & \\
\hline 6434.56 & [Ar v] & 1.86 & 1.32 & 1.24 & 1.19 & & & \\
\hline
\end{tabular}


S. Hyung et al.: P-I Model of PN Hu 1-2, Online Material $p 4$

Table 3. continued.

\begin{tabular}{|c|c|c|c|c|c|c|c|c|}
\hline \multirow[b]{2}{*}{$\lambda_{\mathrm{obs}}$} & \multirow[b]{2}{*}{ Ion } & \multicolumn{3}{|c|}{ North } & \multirow[b]{2}{*}{$I(B)$} & \multicolumn{3}{|c|}{ Center } \\
\hline & & $I(2001)$ & $I(R+B)$ & $I(R)$ & & $I(R+B)$ & $I(R)$ & $I(B)$ \\
\hline 6526.80 & $\mathrm{He}_{\mathrm{II}}$ & & 0.41 & 0.41 & & & & \\
\hline 6527.25 & {$[\mathrm{~N} \mathrm{II}]$} & & & & 0.30 & & & \\
\hline 6548.22 & {$[\mathrm{~N} \mathrm{II}]$} & 40.98 & 58.96 & 70.89 & 27.52 & 71.76 & 78.81 & 60.12 \\
\hline 6559.92 & He II & & 10.88 & 10.85 & 10.95 & 14.72 & 16.66 & 11.51 \\
\hline 6562.81 & $\mathrm{H} \alpha$ & 281.4 & 268.1 & 275.6 & 247.4 & 334.7 & 349.4 & 310.4 \\
\hline 6583.52 & {$[\mathrm{~N}$ II $]$} & 136.3 & 171.1 & 213.0 & 60.49 & 241.7 & 256.3 & 217.4 \\
\hline 6678.18 & $\mathrm{He}_{\mathrm{I}}$ & 2.41 & 2.44 & 3.05 & 0.77 & & & \\
\hline 6682.91 & He II & & 0.50 & 0.53 & 0.43 & & & \\
\hline 6716.67 & [S II] & 4.78 & 5.42 & 6.21 & 3.25 & 6.17 & 6.57 & 5.50 \\
\hline 6730.72 & [S II] & 7.47 & 9.42 & 11.02 & 5.11 & 11.92 & 12.05 & 11.70 \\
\hline 6890.37 & $\mathrm{He}_{\text {II }}$ & 0.97 & 0.75 & 0.68 & 0.56 & 0.76 & & \\
\hline 7005.27 & [Ar v] & 4.16 & 2.69 & 2.58 & 1.86 & & & \\
\hline 7064.85 & $\mathrm{He}_{\mathrm{I}}$ & 4.08 & 5.10 & 6.07 & 2.44 & 6.37 & 6.61 & 5.98 \\
\hline 7135.82 & [Ar III] & 9.63 & 10.96 & 12.15 & 7.70 & 11.81 & 12.10 & 11.34 \\
\hline 7170.36 & [Ar Iv] & & 0.28 & 0.32 & 0.28 & & & \\
\hline 7177.32 & He II & & 0.58 & 0.59 & 0.54 & & & \\
\hline 7237.31 & [Ar Iv] & & 0.37 & 0.38 & 0.49 & 0.37 & & \\
\hline 7262.72 & [Ar IV] & & 0.31 & 0.31 & 0.30 & & & \\
\hline 7281.33 & $\mathrm{He}_{\mathrm{I}}$ & & 0.66 & 0.81 & 0.25 & & & \\
\hline 7319.00 & [O II] & 4.55 & & & & 4.24 & 5.75 & 1.66 \\
\hline 7330.14 & [O II $]$ & 3.97 & & & & 3.18 & 2.93 & 3.58 \\
\hline 7529.66 & {$[\mathrm{Cl}$ IV $]$} & & 0.46 & 0.48 & 0.43 & 0.48 & 0.45 & 0.53 \\
\hline 7592.39 & He II & 1.48 & 1.14 & 1.07 & 1.26 & 1.10 & 0.95 & 1.35 \\
\hline 7751.01 & [Ar III] & 2.67 & 2.18 & 2.60 & 1.05 & & & \\
\hline 8045.50 & {$[\mathrm{Cl}$ IV $]$} & 0.90 & 0.87 & 0.86 & 0.88 & 0.84 & 0.80 & 0.91 \\
\hline 8236.38 & He II & 2.29 & 1.46 & 1.55 & 1.20 & & & \\
\hline 8374.37 & $\mathrm{H}_{\mathrm{I}}$ & & 0.21 & & & & & \\
\hline 8391.62 & $\mathrm{H}_{\mathrm{I}}$ & & 0.27 & & & & & \\
\hline 8413.17 & $\mathrm{H}_{\mathrm{I}}$ & & 0.31 & & & & 0.23 & \\
\hline 8467.17 & $\mathrm{H}_{\mathrm{I}}$ & & 0.49 & & & & & \\
\hline 8502.38 & $\mathrm{H} \mathrm{I}_{\mathrm{I}}$ & & 0.58 & & & & 0.53 & \\
\hline 8543.86 & $\mathrm{H}_{\mathrm{I}}$ & & 0.74 & 0.55 & 0.68 & & 0.61 & \\
\hline 8578.85 & {$[\mathrm{Cl} \mathrm{II}]$} & & 0.16 & & & & & \\
\hline 8598.14 & $\mathrm{H}_{\mathrm{I}}$ & 0.74 & & & & & & \\
\hline 8664.57 & $\mathrm{H} \mathrm{I}_{\mathrm{I}}$ & 0.87 & 0.88 & 0.94 & 0.71 & 0.75 & 0.75 & 0.75 \\
\hline 8750.25 & $\mathrm{H}_{\mathrm{I}}$ & & 0.65 & & & & & \\
\hline 8862.53 & $\mathrm{H}_{\mathrm{I}}$ & & 0.82 & & & & & \\
\hline 9014.60 & $\mathrm{H}_{\mathrm{I}}$ & 1.39 & 0.98 & & & & & \\
\hline 9069.04 & [S III] & 12.26 & 13.38 & 14.14 & 11.26 & 14.86 & 13.87 & 16.50 \\
\hline 9227.98 & $\mathrm{He}_{\mathrm{I}}$ & 1.73 & 1.61 & 1.77 & 1.84 & 1.71 & 1.69 & 1.74 \\
\hline 9344.09 & $\mathrm{He}_{\mathrm{I}}$ & 0.47 & 0.42 & & & & & \\
\hline 9530.59 & [S III] & 25.24 & 21.54 & 24.67 & 12.98 & 36.97 & 33.48 & 42.72 \\
\hline 9545.96 & $\mathrm{H} \mathrm{I}_{\mathrm{I}}$ & 1.75 & 0.94 & 1.02 & 0.54 & & & \\
\hline 9547.26 & $\mathrm{H}_{\mathrm{I}}$ & & & & & 1.80 & 1.97 & 1.53 \\
\hline 10048.95 & $\mathrm{H}_{\mathrm{I}}$ & 2.95 & 2.27 & 2.20 & 1.66 & & & \\
\hline 10123.16 & He II & 15.25 & 9.78 & 11.22 & 5.86 & & & \\
\hline
\end{tabular}

See Hyung (1994) for the identifications and references therein. Interstellar extinction-corrected intensities are all given on the scale of $I(\mathrm{H} \beta)=$ $100(C=0.60)$. $I(2001)$ : intensities of the 2001 Aug observation (at North); $I(R)$ and $I(B)$ are intensities from the red and blue-shifted line profiles, respectively, while $I(R+B)$ are intensities combined from both these profiles. 